Western University

Scholarship@Western

Oncology Publications

Oncology Department

$5-2008$

\title{
Quality assurance of radiation therapy planning systems: current status and remaining challenges.
}

Jacob Van Dyk

The University of Western Ontario, vandyk@uwo.ca

Follow this and additional works at: https://ir.lib.uwo.ca/oncpub

Part of the Medical Biophysics Commons, and the Oncology Commons

Citation of this paper:

Van Dyk, Jacob, "Quality assurance of radiation therapy planning systems: current status and remaining challenges." (2008). Oncology Publications. 134.

https://ir.lib.uwo.ca/oncpub/134 


\title{
QUALITY ASSURANCE OF RADIATION THERAPY PLANNING SYSTEMS: CURRENT STATUS AND REMAINING CHALLENGES
}

\author{
JACOB VAN DYK, M.SC.*广 \\ * Department of Physics and Engineering, London Regional Cancer Program/London Health Sciences Centre, London, ON, Canada; and \\ ${ }^{\dagger}$ Departments of Oncology, Medical Biophysics, Diagnostic Imaging and Nuclear Medicine, and Physics and Astronomy, \\ University of Western Ontario, London, ON, Canada
}

\begin{abstract}
Computerized radiation therapy planning systems (RTPSs) are pivotal for treatment planning. The acceptance, commissioning, and quality control of RTPSs are uniquely complex and are described in the American Association of Physicists in Medicine Task Group Report 53 (1998) and International Atomic Energy Agency Technical Report Series No. 430 (2004). The International Atomic Energy Agency also developed a document and data package for use by vendors and purchasers to aid with acceptance testing of RTPSs. This document is based on International Electrotechnical Commission standard 62083 (2000) and describes both "type" tests to be performed in the factory and "site" tests to be performed in the clinic. The American Association of Physicists Task Group Report 67 described benchmark tests for the validation of dose calculation algorithms. Test data are being produced with the backing of the U.S. National Cancer Institute. However, significant challenges remain. Technology keeps evolving rapidly, thus requiring new quality assurance (QA) procedures. Intensity-modulated radiation therapy with its use of inverse optimization has added a new dimension to QA, because the results are not intuitively obvious. New technologies such as real-time ultrasound guidance for brachytherapy, TomoTherapy, and Cyberknife, require their own specialized RTPSs with unique QA requirements. On-line imaging allows for the generation of dose reconstructions using image warping techniques to determine the daily dose delivered to the patient. With increasing computer speeds, real-time reoptimization of treatment plans will become a reality. Gating technologies will require four-dimensional dose calculations to determine the actual dose delivered to tissue voxels. With these rapidly changing technologies, it is essential that a strong QA culture is invoked in every institution implementing these procedures and that new protocols are developed as a part of the clinical implementation process. (C) 2008 Elsevier Inc.
\end{abstract}

Quality assurance, Treatment planning, Radiation therapy planning systems.

\section{INTRODUCTION}

A tremendous evolution (some would say a revolution) in radiation oncology has occurred in recent years. These rapid changes and enhancements have resulted from developments in computer technology, which have allowed advancements in diagnostic imaging and radiation therapy delivery capabilities. The result is that imaging using various procedures (e.g., computed tomography, positron emission tomography, single photon emission tomography, magnetic resonance imaging, ultrasonography) is much more readily available as a part of the radiation therapy planning process. In addition, enhancements in computer-controlled dose delivery, along with the use of multileaf collimators has allowed for both static (step-and-shoot) and dynamic intensity-modulated radiation therapy (IMRT). These new technologies have allowed for more controlled dose delivery with greater dose gradients and tighter margins. The net effect is that radiation oncologists are able to prescribe greater doses while maintaining normal tissue toxicities at acceptable levels. Central to the application of these new technologies is the radiation therapy planning system (RTPS).

A review of the historical development of RTPSs can be found in a recent chapter by Van Dyk (1). The modern RTPS allows for the use of images from various imaging modalities to aid in the definition of target volumes. It has more
Reprint requests to: Jacob Van Dyk, M.Sc., Department of Physics and Engineering, London Regional Cancer Program/London Health Sciences Centre, 790 Commissioners Rd. E., London, ON N6A 4L6 Canada. Tel: (519) 685-8607; Fax: (519) 685-8658; E-mail: jake.vandyk@lhsc.on.ca

Conflict of interest: J. Van Dyk has a license agreement with Modus Medical Devices, Inc. for the development and sale of QUASAR Phantoms.
Acknowledgments - This report is based on the work of various national and international committees and task groups dealing with quality assurance of radiotherapy planning systems, with the author being a member of a number of these. The author acknowledges the expertise provided by the members of these working groups in developing these reports and in providing a basis for this report.

Received Feb 16, 2007, and in revised form April 17, 2007. Accepted for publication April 18, 2007. 
sophisticated calculation algorithms, providing more accurate dose calculation capabilities, especially for the small beams associated with IMRT delivery techniques. Physical or dynamic wedge calculation capabilities are provided. Automated optimization routines used in conjunction with inverse planning are available to help define the multileaf collimator delivery configurations. More sophisticated dose distribution evaluation tools are integral to automated optimization and plan evaluation. These include the use of dose-volume constraints, display of dose-volume histograms, and the application of biologically related endpoints such as tumor control probability, normal tissue complication probability, and the equivalent uniform dose. For plan delivery verification, doses can be reconstructed for specific phantom configurations, and digitally reconstructed radiographs can be produced to compare with the portal images.

\section{NATIONAL AND INTERNATIONAL REPORTS ON RTPSs}

Early reports on the commissioning and quality assurance (QA) of TPSs are relatively sparse. Perhaps the forerunner of these was a study in 1980 by McCullough and Krueger (2). The first committee findings from Canada was reported by Van Dyk et al. (3). An early international report on RTPSs was published in 1987 by the International Commission on Radiation Units and Measurements in its Report 42 (4). That report provided a detailed description of the state-ofthe art of RTPSs of that time; however, only two pages were devoted to QA. The American Association of Physicists in Medicine (AAPM) Task Group Report 65 (5) provided a detailed description of dose calculation algorithms used by RTPSs, especially in relation to tissue inhomogeneity corrections. Recent years have seen various reports by national and international organizations that have made recommendations regarding the commissioning and QA of RTPSs. In 1998, the AAPM published Task Group report 53 (6), giving guidelines for users and vendors on QA for radiation therapy planning. In 2000, the International Electrotechnical Commission produced report No. 62083 (7), identifying the safety requirements for manufacturers of RTPSs. In 2004, both the International Atomic Energy Agency (IAEA) (8) and the European Society of Therapeutic Radiation Oncology (9) published reports on the commissioning and QA of RTPSs. Furthermore, the IAEA has recently developed a report for acceptance testing of RTPSs (10). In 2006, The Netherlands Commission of Radiation Dosimetry also produced a report on QA of RTPSs (11). The IAEA Technical Report Series No. 430 (TRS-430) (8) is perhaps the most comprehensive of all these reports, because it attempted to be a guide for the entire gamut of RTPSs found worldwide.

\section{IAEA TRS-430 guidelines}

The IAEA TRS- 430 begins by providing a rationale for QA of RTPSs by describing significant treatment errors that have occurred because of the inappropriate development of QA procedures in the clinic. Although this report is intended as a generic guide for the commissioning and QA of RTPSs, it does not provide a simple or unique protocol for these tasks because (1) internationally, a wide variety of treatment machine capabilities exists, ranging from simple ${ }^{60} \mathrm{Co}$ machines to complex treatment machines with multileaf collimators and the possibility of using IMRT; (2) a wide variety of treatment procedures are in place that are dependent on institutional resources, patient imaging availability for treatment planning, and treatment machine capabilities; and (3) commercial RTPSs have a wide diversity of capabilities, ranging from relatively simple two-dimensional systems to comprehensive three-dimensional treatment planning capabilities that make full use of three-dimensional image data sets, possibly from various imaging modalities. To provide guidance for this very large scope of capabilities, the TRS- 430 provides a comprehensive process that should be useful to every institution providing radiation therapy. The report provides specific examples of the kinds of tests that need to be performed for both commissioning and quality control (QC) purposes.

The IAEA TRS-430 does not address issues related to acceptance testing in adequate detail. Although acceptance testing is well-defined and a standard process for the purchase of other radiation therapy equipment, it is not nearly as straightforward for RTPSs. This process is complicated because the clinical implementation of an RTPS requires the user to obtain, usually by measurement, very specific data needed by the RTPS for proper functioning of the dose calculation algorithm for the specific radiotherapy machines used to treat patients in the user's clinic. To address this issue, the IAEA has developed a new report (10) that is complete and was published in early 2007. That report used as a guiding document the International Electrotechnical Commission specifications and safety requirements, Standard 62083 for RTPSs (7), which was published in 2000 and specifically aimed at manufacturers. The IAEA acceptance protocol requires vendors to perform and document a series of "type" tests using beam commissioning data supplied by the IAEA. The beam data and added tests were based on concepts originally developed in the AAPM Report 55 (12) and later updated by Venselaar and Welleweerd (13). Using the beam data provided with the IAEA acceptance report, the user selects a subset of the vendor "type" tests and performs "site" tests to ensure that the software complies with the standards defined in the report.

\section{Works in progress}

The IAEA has initiated an additional document to be used as a guide for the commissioning of RTPSs, although this document is primarily intended to help clinics in the developing world, largely with simpler RTPSs. Meanwhile, using the recommendations from AAPM Task Group report 53, the AAPM Task Group report 67 (unpublished data: Bayouth J, Followill D, Fraass B, et al. AAPM Radiation Therapy Committee Task Group 67: Benchmark datasets for photon beams. 2005) described the development of a series of benchmark tests for the validation of dose calculation algorithms of RTPSs. The measured data for these benchmark tests are 
now being produced with the financial support of the U.S. National Cancer Institute.

\section{REMAINING CHALLENGES}

Although progress in the development of these documents, protocols, and standards is certainly a dramatic improvement over work done in previous decades, significant challenges remain. Technology keeps evolving at such a rapid rate that it is difficult to maintain up-to-date, routine, and documented QA procedures and protocols. The following highlights some of the outstanding challenges. Some of these challenges overlap between fundamental research to develop procedures to improve the quality of treatment and actually addressing $\mathrm{QA}$ and QC issues related to the implementation of new procedures and technologies.

\section{Plan optimization parameters}

Modern RTPSs provide automated plan optimization capabilities using objective functions that aid in the determination of the quality of the plan. Objective functions contain information about the desired and actual dose distribution. The form of the objective function tends to be unique to each commercial system. Included in the objective function are quantities that aid in determining the importance of one endpoint vs. another. These quantities are often known as "importance" factors or "weighting" factors or "penalty" factors. As a part of the planning process, the user needs to define the dose-volume constraints for each structure of interest, whether tumor or organ at risk. In addition, the treatment planner needs to determine the values for the importance/weighting/penalty factors. The choice of values for these factors is dependent on the treatment site, tumor size and location, and normal tissue type. The present practice is for each clinic to develop its own experience on the values for these factors based on practical experience for individual treatment sites. The result is that the actual implementation of automated optimization procedures is based on the very subjective choice of these factors. One of the remaining challenges for the medical physic community is to determine some form of standardization of both objective functions and the relevant importance/weighting/penalty factors. It would then be possible to generate some class solutions for the use of these factors that can be applied to some generic clinical situations.

\section{Optimization in presence of uncertainties}

Recent years have seen a significant number of publications addressing the uncertainties associated with the radiation therapy process, including setup, geometric, and organ motion uncertainties. Interest is growing in accounting for these uncertainties in the optimization process (14-18), because of recognition that an optimized plan developed without accounting for uncertainties could be quite different from one developed that did account for the uncertainties. Once implemented, these algorithms will require special
QA procedures to test for their capabilities, limitations, and proper functioning.

\section{Intensity-modulated radiation therapy $Q A$}

Intensity-modulated radiation therapy, with its use of inverse optimization, has added a new dimension to QA, because the results are no longer intuitively obvious, and manual or simple checks of the results are not possible. Consequently, individualized patient plans must be recalculated for phantom geometries and corresponding measurements performed on the phantom for patient-specific treatment procedures. None of the publications described in the present report addressed the QA and QC issues adequately for IMRT planning. Recent trends are moving toward independent software packages that can calculate the monitor units using the IMRT delivery configurations provided by the RTPS but using entirely different software. Because this software tends to be less sophisticated than the software used by the RTPS that developed the original plan, under certain conditions, discrepancies are likely to result between the original monitor unit calculations done on the RTPS and those done by the QC software. The user must make an educated rationalization as to whether the results are acceptable or whether the difference is significant and needs additional review. Thus, two issues evolve from this process. The first is that new QA techniques must be developed to evaluate the software that performs the secondary checks. The second is that the criteria of acceptability between the results of the primary software calculations and the secondary software calculations need to be developed such that a consistent and meaningful assessment is possible of the comparison of these results.

For treatment planning software associated with specialized treatment technologies (e.g., helical TomoTherapy, Cyberknife), no third-party QA software exists. Does this mean that users must perform patient-specific dose delivery QA measurements indefinitely or can alternative QC techniques be developed? Thus, the challenge is to develop time-efficient QA procedures for these specialized technologies.

\section{Plan evaluation and radiobiological models}

Modern RTPSs are also providing new plan evaluation and optimization capabilities such as dose-volume histogram comparisons and radiobiological evaluation. To date, the clinical application of radiobiological models remains controversial, because a general mistrust exists of their clinical relevance and a clear understanding of their capabilities and limitations has not been achieved (19). However, commercial vendors of RTPSs are providing radiobiological models that allow users to apply them to clinical situations. The challenge is that educational materials are needed for users of these systems to describe the capabilities and limitations of both the models themselves and the corresponding uncertainties in the parameters used in these models, because they are generally derived from limited clinical data. One approach used by some is to use a radiobiological tumor control probability and normal tissue complication probability calculation for patients undergoing treatment, not as a means of optimizing 
the treatment, but rather as a QA tool. If the resulting tumor control probability, or normal tissue complication probability, is of concern, this would require follow-up. Thus, the radiobiologic model, at least in its early phase of clinical implementation, is not the primary calculation determining the treatment technique but becomes a QA check. It is only after sufficient clinical evidence is available that radiobiological models can provide a prediction of treatment outcome accurate enough that they should come into routine clinical practice.

\section{Dose reconstruction}

Daily on-line imaging allows for the generation of dose reconstructions using image or dose warping techniques to determine the actual daily dose delivered to specific voxels within the tumor and organs at risk (20-22). Furthermore, as the speed of computers improves, real-time reoptimization of treatment plans, using the anatomy of the day, will become a reality in the future. Again, the challenge will be to assure the users that both the daily imaging system and the corresponding dose reconstruction and reoptimization algorithms behave as intended, especially when done in a real-time mode of operation.

\section{Four-dimensional treatment}

At present, four-dimensional computed tomography and beam gating technologies determine the specified times during the breathing cycle when the beam is to be turned on or off. The research challenge is to ensure a consistent correlation between tumor motion and external fiducials or the references used to trigger the beam gating system. In addition, to obtain a sense of the true dose delivered to both the target and the organs at risk, gating technologies will require fourdimensional dose calculations to be performed during the parts of the breathing cycle in which the beam is on. Furthermore, QA procedures will need to be developed for both the four-dimensional dose calculation procedures and the actual gated delivery of the radiation dose.

\section{Phantoms and QA tools}

As a result of the increasing complexity of the radiation therapy process, new and more specialized QA and QC procedures are being developed. This will require new QA tools, new phantoms, and new analysis procedures. With highly shaped dose distributions, the trend is toward multidimensional measurement techniques with two-dimensional detector arrays and three-dimensional gel dosimetry (for a summary see Van Dyk [23]). Furthermore, the use of gating and dose delivery techniques to account for breathing motion requires the use of phantoms that include a time component. Although technologies and QA tools are being developed, no consistent or cohesive approach is yet available for quality assessment of these new technologies. Thus, the challenge remains for improved QA and QC tools that are relatively inexpensive, relatively easy to use, and relatively universal to apply.

\section{CONCLUSION}

The RTPS is at the hub of the overall radiation therapy process. This report provides a review of the documents produced by various working groups, both national and international, associated with QA of RTPSs. With the rapid development and on-going changes in imaging and radiation therapy technologies, QA and QC procedures require constant redevelopment and evolution. Future directions are considered and challenges outlined. With these rapidly changing technologies, it is essential that a strong QA culture is invoked in every institution implementing these new and advanced procedures and that new protocols are developed as a part of the clinical implementation process.

\section{REFERENCES}

1. Van Dyk J. Quality assurance. In: Khan FM, editor. Treatment planning in radiation oncology. 2nd ed. Philadelphia: Lippincott, Williams \& Wilkins; 2007. p. 98-115.

2. McCullough EC, Krueger AM. Performance evaluation of computerized treatment planning systems for radiotherapy: External photon beams. Int J Radiat Oncol Biol Phys 1980; 6:1599-1605.

3. Van Dyk J, Barnett RB, Cygler JE, et al. Commissioning and quality assurance of treatment planning computers. Int J Radiat Oncol Biol Phys 1993;26:261-273.

4. International Commission on Radiation Units and Measurements. ICRU report 42: Use of computers in external beam radiotherapy procedures with high-energy photons and electrons. Bethesda: International Commission on Radiation Units and Measurements; 1987

5. Papanikolaou N, Battista JJ, Boyer AL, et al. AAPM Report 85: Tissue inhomogeneity corrections for megavoltage photon beams-Report by Task Group 65 of the Radiation Therapy Committee of the American Association of Physicists in Medicine. Madison: Medical Physics Publishing; 2004.
6. Fraass B, Doppke K, Hunt M, et al. American Association of Physicists in Medicine Radiation Therapy Committee Task Group 53: Quality assurance for clinical radiotherapy treatment planning. Med Phys 1998;25:1773-1829.

7. International Electrotechnical Commission (IEC). Medical electrical equipment-Requirements for the safety of radiotherapy treatment planning systems. IEC 62083 (2000-11). Geneva: International Electrotechnical Commission; 2000.

8. Van Dyk J, Rosenwald J-C, Fraass B, et al. Commissioning and quality assurance of computerized planning systems for radiation treatment of cancer: IAEA TRS-430. Vienna: International Atomic Energy Agency; 2004.

9. Mijnheer B, Olszewska A, Fiorino C, et al. Quality assurance of treatment planning systems: Practical examples of non-IMRT photon beams. Brussels: European Society of Therapeutic Radiation Oncology; 2004.

10. Ibbott G, Schmidt R, Van Dyk J, et al. Protocol for specification and acceptance testing of radiation treatment planning systems. IAEA TECDOC Series No. 1540. Vienna: International Atomic Energy Agency; 2007. 
11. Bruinvis IAD, Keus RB, Lenglet WJM, et al. Quality assurance of 3-D treatment planning systems for external photon and electron beams: Report 15 of The Netherlands Commission on Radiation Dosimetry. Delft, The Netherlands: The Netherlands Commission on Radiation Dosimetry; 2006.

12. Miller DW, Bloch PH, Cunningham JR, et al. Radiation treatment planning dosimetry verification: AAPM Report Number 55. New York: American Institute of Physics; 1995.

13. Venselaar J, Welleweerd H. Application of a test package in an intercomparison of the photon dose calculation performance of treatment planning systems used in a clinical setting. Radiother Oncol 2001;60:203-213.

14. Wong E, Van Dyk J, Battista JJ, et al. Uncertainty analysis: A guide to optimization in radiation treatment planning. In: Leavitt DD, Starkschall G, editors. Proceedings of the XIIth International Conference on the use of computers in radiotherapy. Madison, WI: Medical Physics Publishing; 1997. p. 259-261.

15. Unkelbach J, Oelfke U. Incorporating organ movements in inverse planning: assessing dose uncertainties by bayesian inference. Phys Med Biol 2005;50:121-139.

16. Unkelbach J, Oelfke U. Inclusion of organ movements in IMRT treatment planning via inverse planning based on probability distributions. Phys Med Biol 2004;49:4005-4029.
17. Yang J, Mageras GS, Spirou SV, et al. A new method of incorporating systematic uncertainties in intensity-modulated radiotherapy optimization. Med Phys 2005;32:2567-2579.

18. McShan DL, Kessler ML, Vineberg K, et al. Inverse plan optimization accounting for random geometric uncertainties with a multiple instance geometry approximation (MIGA). Med Phys 2006;33:1510-1521.

19. Moiseenko V, Deasy JO, Van Dyk J. Radiobiological modeling for treatment planning. In: Van Dyk J, editor. The modern technology of radiation oncology: a compendium for medical physicists and radiation oncologists. Madison, WI: Medical Physics Publishing; 2005. p. 185-220.

20. Schaly B, Kempe JA, Bauman GS, et al. Tracking the dose distribution in radiation therapy by accounting for variable anatomy. Phys Med Biol 2004;49:791-805.

21. Langen KM, Meeks SL, Poole DO, et al. The use of megavoltage CT (MVCT) images for dose recomputations. Phys Med Biol 2005;50:4259-4276.

22. Rosu M, Chetty IJ, Balter JM, et al. Dose reconstruction in deforming lung anatomy: dose grid size effects and clinical implications. Med Phys 2005;32:2487-2495.

23. Van Dyk J. Advances in modern radiation therapy. In: Van Dyk J, editor. The modern technology of radiation oncology: a compendium for medical physicists and radiation oncologists. Madison, WI: Medical Physics Publishing; 2005. p. 1-29. 